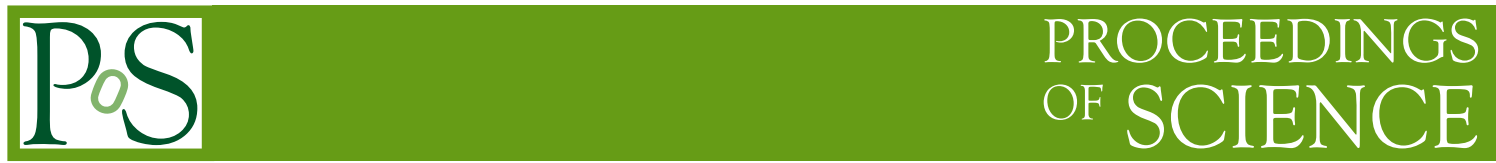

\title{
The Square Kilometre Array
}

\section{T. Joseph W. Lazio*†}

Naval Research Laboratory and Square Kilometre Array Program Development Office, 4555

Overlook Ave. SW, Washington, DC 20375-5351 USA

E-mail: laziodskatelescope.org

The Square Kilometre Array (SKA) is intended as the next-generation radio telescope and will address fundamental questions in astrophysics, physics, and astrobiology. The international science community has developed a set of Key Science Programs: (1) Emerging from the Dark Ages and the Epoch of Reionization, (2) Galaxy Evolution, Cosmology, and Dark Energy, (3) The Origin and Evolution of Cosmic Magnetism, (4) Strong Field Tests of Gravity Using Pulsars and Black Holes, and (5) The Cradle of Life/Astrobiology. In addition, there is a design philosophy of "exploration of the unknown," in which the objective is to keep the design as flexible as possible to allow for future discoveries. Both a significant challenge and opportunity for the SKA is to obtain a significantly wider field of view than has been obtained with radio telescopes traditionally. Given the breadth of coverage of cosmic magnetism and galaxy evolution in this conference, I highlight some of the opportunities that an expanded field of view will present for other Key Science Programs.

Panoramic Radio Astronomy: Wide-field 1-2 GHz research on galaxy evolution - PRA2009 June 02 - 052009

Groningen, the Netherlands

\footnotetext{
* Speaker.

${ }^{\dagger}$ Basic research in radio astronomy at the NRL is supported by 6.1 Base funding.
} 


\section{Introduction}

In the $20^{\text {th }}$ Century, we discovered our place in the Universe. We learned that it was much bigger than we imagined and much more exotic. Beyond our Milky Way, the Universe is filled with galaxies - each their own island universe. They range in size from dwarf galaxies barely able to survive near their larger neighbors to giant elliptical galaxies, orders of magnitudes larger than the Milky Way. These galaxies of stars also contain a multitude of other components including gas with a wide range of temperatures; compact objects including white dwarfs, neutron stars, and black holes; and planets. Over the course of the century, black holes moved from a theoretical curiosity to a well-recognized endpoint of stellar evolution and a likely fundamental component of the centers of galaxies, with the potential to power immense jets of relativistic particles that affect their surroundings. By the end of the century, we were beginning to unveil the basic structure and processes of the Universe in which these objects are embedded, including evidence of its origin and the still mysterious properties of dark matter and dark energy.

Our probes of the Universe have expanded dramatically as well. Electromagnetic radiation has been detected from celestial objects at frequencies below $1 \mathrm{MHz}(\lambda \sim 300 \mathrm{~m})$ to energies exceeding $1 \mathrm{TeV}$. Moreover, the range of possible signals has expanded beyond just electromagnetic radiation. Cosmic rays rain down on the Earth, some with energies approaching those of macroscopic objects. Gravitational radiation has been detected indirectly, and numerous potential classes of sources have been suggested, with the expectation that the Earth is awash in gravitational waves. Neutrinos have been detected from both the Sun and supernova 1987A, and many of the processes that generate high energy cosmic rays should also produce a spectrum of high-energy neutrinos.

In the $21^{\text {st }}$ Century, we seek to understand the Universe we inhabit. To do so will require a suite of powerful new instruments, on the ground and in space, operating across the entire electromagnetic spectrum and for multiple decades. Observations at centimeter- to meter wavelengths have provided deep insight to a wide range of phenomena ranging from the solar system to the most distant observable celestial emission. This long and rich record of important discoveries in the radio spectrum, including 3 Nobel prizes, has been possible since many of the relevant physical phenomena can only be observed, or understood best, at these wavelengths. These phenomena include the cosmic microwave background (CMB), quasars, pulsars, gravitational waves, astrophysical masers, magnetism from planets through galaxies, the ubiquitous jets from black holes and other objects, and the spatial distribution of hydrogen gas, the predominant baryonic constituent of the Universe. Moreover, through the invention of aperture synthesis, also recognized by the Nobel committee, radio astronomy has reached unprecedented levels of imaging resolution and astrometric precision, providing the fuel for further discovery.

With only a handful of exceptions, radio telescopes and arrays have been limited to apertures of about $10^{4} \mathrm{~m}^{2}$, constraining, for instance, studies of the 21-centimeter hydrogen emission to the nearby Universe $(z \sim 0.2)$ [4]. Contemporaneous with the astronomical discoveries in the latter half of the $20^{\text {th }}$ Century have been technological developments that offer a path to substantial improvements in future radio astronomical measurements. Among the improvements are mass production of centimeter-wavelength antennas enabling apertures potentially 100 times larger than previously available, fiber optics for the transmission of large volumes of data, high-speed digital signal processing hardware for the acquisition and analysis of the signals, and computational improvements 


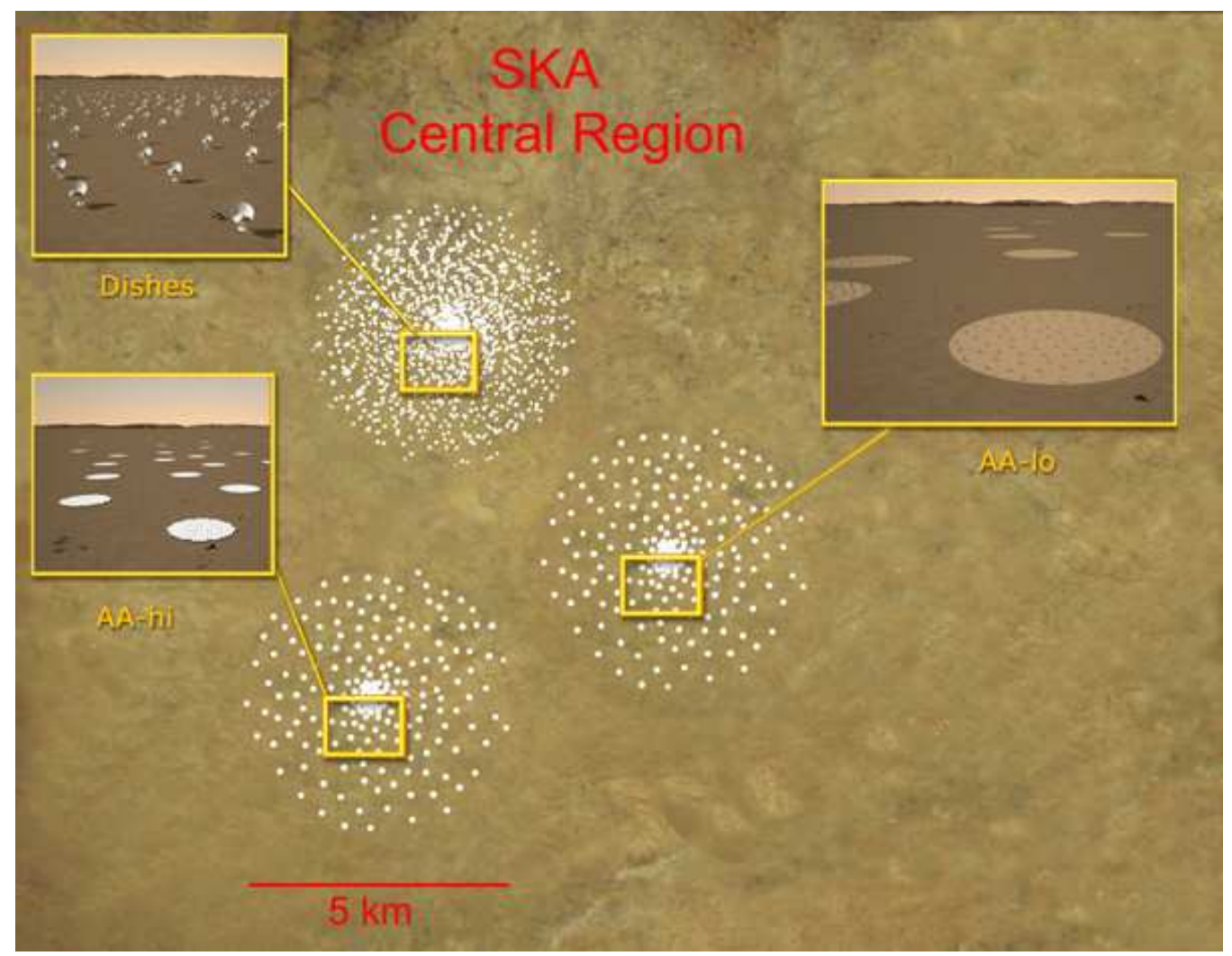

Figure 1: An artist's impression of the core of the SKA illustrating the various technologies over the frequency range $70 \mathrm{MHz}$ to $10 \mathrm{GHz}$. All of these technologies would enable various levels of wide-field imaging.

leading to massive processing and storage. These new technologies, combined with dramatically improved survey speeds and the other advances, can open up an enormous expanded volume of discovery space, providing access to many new celestial phenomena and structures, including 3dimensional mapping of the web of hydrogen gas through much of cosmic history $(z \sim 2)$.

The realization that radio astronomy was on the doorstep of a revolutionary age of scientific breakthrough has led the international community to investigate this opportunity in great detail over the last decade. That coordinated effort, involving a significant fraction of the world's radio astronomers and engineers, has resulted in the Square Kilometre Array (SKA) Program (Figure 1), an international roadmap for the future of radio astronomy over the next two decades and one for which access to a wide field of view is an integral part of the science.

From its inception, development of the SKA Program has been a global endeavor. In the early 1990s, there were multiple, independent suggestions for a "large hydrogen telescope." It was recognized that probing the fundamental baryonic component of the Universe much beyond the local Universe would require a substantial increase in collecting area. The IAU established a working group in 1993 to begin a worldwide study of the next generation radio observatory. Since that time, the effort has grown to comprise 19 countries and more than 50 institutes, including about 200 scientists and engineers. 


\section{Key SKA Science}

Over the past several years, there has been extensive activity related to developing a detailed science case for the SKA, culminating in the SKA Science Book [4]. Highlighting the SKA Science Case are Key Science Projects (KSPs), which represent unanswered questions in fundamental physics, astrophysics, and astrobiology. Furthermore, each of these projects has been selected using the criterion that it represents science that is either unique to the SKA or in which the SKA will provide essential data for a multi-wavelength analysis [6]. The KSPs are

Emerging from the Dark Ages and the Epoch of Reionization The ionizing ultra-violet radiation from the first stars and galaxies produced a fundamental change in the surrounding intergalactic medium, from a nearly completely neutral state to the nearly completely ionized Universe in which we live today. The most direct probe of this Epoch of Re-ionization (EoR), and of the first large-scale structure formation, will be obtained by imaging neutral hydrogen and tracking the transition of the intergalactic medium from a neutral to ionized state. Moreover, as the first galaxies and AGN form, the SKA will provide an unobscured view of their gas content and dynamics via observations of highly redshifted, low-order molecular transitions (e.g., CO).

Galaxy Evolution, Cosmology, and Dark Energy Hydrogen is the fundamental baryonic component of the Universe. The SKA will have sufficient sensitivity to the 21-cm hyperfine transition of $\mathrm{H}$ I to detect galaxies to redshifts $z>1$. One of the key questions for $21^{\text {st }}$ Century astronomy is the assembly of galaxies; the SKA will probe how galaxies convert their gas to stars over a significant fraction of cosmic time and how the environment affects galactic properties. Simultaneously, baryon acoustic oscillations (BAOs), remnants of early density fluctuations in the Universe, serve as a tracer of the early expansion of the Universe. The SKA will assemble a large enough sample of galaxies to measure BAOs as a function of redshift to constrain the equation of state of dark energy.

The Origin and Evolution of Cosmic Magnetism Magnetic fields likely play an important role throughout astrophysics, including in particle acceleration, cosmic ray propagation, and star formation. Unlike gravity, which has been present since the earliest times in the Universe, magnetic fields may have been generated essentially $a b$ initio in galaxies and clusters of galaxies. By measuring the Faraday rotation toward large numbers of background sources, the SKA will track the evolution of magnetic fields in galaxies and clusters of galaxies over a large fraction of cosmic time. The SKA observations also will seek to address whether magnetic fields are primordial and dating from the earliest times in the Universe or generated much later by dynamo activity.

Strong Field Tests of Gravity Using Pulsars and Black Holes With magnetic field strengths as large as $10^{14} \mathrm{G}$, rotation rates approaching $1000 \mathrm{~Hz}$, central densities exceeding $10^{14} \mathrm{~g} \mathrm{~cm}^{-3}$, and normalized gravitational strengths of order 0.4 , neutron stars represent extreme laboratories. Their utility as fundamental laboratories has already been demonstrated through results from observations of a number of objects. The SKA will find many new millisecond pulsars and engage in high precision timing of them in order to construct a Pulsar Timing Array for 
the detection of nanohertz gravitational waves, probing the space-time environment around black holes via both ultra- relativistic binaries (e.g., pulsar-black hole binaries) and pulsars orbiting the central supermassive black hole in the centre of the Milky Way, and probe the equation of state of nuclear matter.

The Cradle of Life The existence of life elsewhere in the Universe has been a topic of speculation for millennia. In the latter half of the $20^{\text {th }}$ Century, these speculations began to be informed by observational data, including organic molecules in interstellar space, and proto-planetary disks and planets themselves orbiting nearby stars. With its sensitivity and resolution, the SKA will be able to observe the centimeter-wavelength thermal radiation from dust in the inner regions of nearby proto-planetary disks and monitor changes as planets form, thereby probing a key regime in the planetary formation process. On larger scales in molecular clouds, the SKA will search for complex prebiotic molecules. Finally, detection of transmissions from another civilization would provide immediate and direct evidence of life elsewhere in the Universe, and the SKA will provide sufficient sensitivity to enable, for the first time, searches for unintentional emissions or "leakage."

In addition to the KSPs listed, and recognizing the long history of discovery at radio wavelengths (pulsars, cosmic microwave background, quasars, masers, the first extrasolar planets, etc.), the international science community also recommended that the design and development of the SKA have "Exploration of the Unknown" as a philosophy. Wherever possible, the design of the telescope is being developed in a manner to allow maximum flexibility and evolution of its capabilities to probe new parameter space (e.g., time-variable phenomena that current telescopes are not well-equipped to detect). This philosophy is essential as many of the outstanding questions of the 2020-2050 era-when the SKA will be in its most productive years-are likely not even known today.

\section{Opportunities for Panoramic SKA Science}

Many of the papers in this volume illustrate far better than I could the opportunities for Panoramic SKA Science, particularly in the areas of cosmic magnetism and galaxy structure and evolution via H I observations. Consequently, and similar to my approach in the conference itself, I shall focus on opportunities for wide-field observations as they concern some of the other SKA KSPs.

\subsection{Emerging from the Dark Ages and the Epoch of Reionization}

The primary focus of this KSP is tracking the transition from the Universe's largely neutral state to its currently nearly completed ionized state. Wide-field observations will be both important and natural as the key observations will be of the highly-redshifted $\mathrm{H}$ I line at frequencies below $200 \mathrm{MHz}$, which will be carried out using dipole-based sparse aperture arrays. Dipoles have fields of view that can exceed $\pi$ sr easily, but the relevant frequencies are below the nominal focus of this conference. 
A potential and important secondary observation that could be conducted near $1 \mathrm{GHz}$, however, would be of the synchrotron radiation from the first galaxies [12]. Copious numbers of massive stars would have likely formed within these first galaxies and then exploded soon thereafter as supernovae. If the interstellar magnetic fields of these galaxies have developed sufficiently, the galaxies will emit synchrotron radiation as a result of cosmic rays accelerated by the supernova remnants from these first massive stars. While it is not yet known if these galaxies will be detectable, the radio-far infrared correlation for star-forming galaxies is now known to hold at least out to a redshift $z \approx 3$ [14]. If it continues to hold to $z \approx 6$, then radio observations would be a powerful means of probing dust-enshrouded first galaxies and wide-field observations would naturally allow for large volumes of the Universe to be sampled quickly.

\subsection{Fundamental Physics Using Observations of Pulsars and Black Holes}

Wide-field capabilities that enable the SKA to access a substantial solid angle will be important for pulsar studies, even though pulsars are point sources so that "panoramic imaging" per se of them is unlikely to be profitable.

Fundamental physics constraints are derived from pulsar observations via long-term timing programs that measure precisely the times of arrival of the pulses. A significant constraint on the utility of radio pulsars is the scarcity of "useful" pulsars. For instance, until recently, the most significant constraints on the nuclear equation of state derived from radio pulsars resulted from the first millisecond pulsar, PSR B1937+21, discovered in the early 1980s. Similarly, many of the tests of theories of gravity and for gravitational wave emission rely on one or a few objects. Recent surveys have begun to demonstrate the potential for vastly increasing the number of radio pulsars and thereby increasing the number of "useful" systems.

Perhaps the best example of the impact of increasing field of view for pulsar surveys is the Parkes Multibeam Survey [10]. By installing a multiple feed horn system on the Parkes antenna, the effective field of view was increased by a factor of 13 . The resulting survey essentially doubled the total number of pulsars. (See also \$3.4.) Future field-of-view expansion technologies (e.g., phased array feeds or dense aperture arrays) coupled with the vastly increased sensitivity of the SKA offer promise for an even larger yield.

The impact of a wide field of view for pulsar timing and monitoring programs is less clear. In principle, a telescope with a sufficiently wide field of view could time multiple pulsars simultaneously, yielding an improved "throughput." In practice, the current estimates of the density on the sky of "useful" pulsars is sufficiently low that only dense aperture arrays are likely to have a field of view that could be large enough to time multiple pulsars simultaneously, except perhaps in special regions of the sky. Moreover, in order to mitigate interstellar propagation effects, timing observations have to be carried out over a relatively large frequency range (e.g., 0.8-3 GHz), wider than what dense aperture arrays are currently thought to be able to achieve.

\subsection{Cradle of Life/Astrobiology}

One of the key assumptions in the search for life elsewhere in the Universe, particularly in searches for life within the solar system, is that other life is likely to be based on carbon chemistry (i.e., "organic"), like life on Earth is. Prime support for this approach is that the vast majority of 
multi-atom molecules in interstellar space contain carbon, including a number of complex organic species [15, 2, 1, and references within]. As the number of atoms increases, the rotational and vibrational transitions tend to shift to lower frequencies, and searches for and studies of complex organic molecules have relied upon observations below $2 \mathrm{GHz}$. Consequently, a wide field-of-view at frequencies around $1 \mathrm{GHz}$ could be quite valuable for conducting surveys of molecular clouds for complex organic molecules; such observations would find a natural complement in ALMA observations.

Direct evidence for life elsewhere in the Universe would be the detection of signals from another technological civilization. Two examples from our own civilization are cell phone transmissions and aeronautical navigation, both of which make use of frequencies around $1 \mathrm{GHz}$, though neither are strong enough to be detectable over interstellar distances (even with the SKA!). More generally, the "waterhole" between 1.4 and $1.7 \mathrm{GHz}$ has been a focus of numerous previous searches for extratestrial transmissions, as it has been argued that any technological civilization capable of trying to communicate over interstellar distances would certainly know about the $\mathrm{H} \mathrm{I}$ line at $1.4 \mathrm{GHz}$ and the $\mathrm{OH}$ lines around $1.7 \mathrm{GHz}$.

One approach for searching for extraterrestial intelligence (SETI) is to monitor a "habstar," a star that might be orbited by a terrestrial planet(s) within the star's habitable zone [16]. Much like pulsar timing, being able to monitor multiple habstars would increase the throughput of SETI observations; the key contrast between pulsar and habstar observations is that the density on the sky of suitable main sequence stars is sufficiently high that most, if not all, fields of view will include more than one habstar.

\subsection{The Dynamic Radio Sky}

A series of discoveries over the past decade have both illustrated and emphasized that the time domain has been explored only poorly at radio wavelengths [3, 7, 9, 11]. Although time resolutions approaching $1 \mathrm{~ns}$ have been achieved [8], typically these have been obtained only on relatively narrow fields of view. The challenge and opportunity for the SKA, and consistent with the "exploration of the unknown" design philosophy, is to obtain both high time resolution and access to a significant solid angle.

Some of these observations might naturally happen in the course of pulsar surveys ( $\$ 3.2)$; indeed, the discovery of rotating radio transients, a new class of radio-emitting neutron stars, resulted from the novel processing of a pulsar survey [11]. Other types of transient surveys and exploration programs might utilize wide fields of view in different manners, however.

We provide two examples to illustrate the potential range of applications of a wide field of view:

1. Extreme scattering events (ESEs) are a class of dramatic flux density variations $(\sim 50 \%)$ of extragalactic sources caused by intervening plasma lenses [5]. The initial surveys for ESEs observed only a relatively small number of the strongest, most compact sources on the sky. Yet within even a modest field of view, if the full field of view can be imaged, are potentially tens to hundreds of sources. A potential ESE search program could be conducted by surveying a significant solid angle with a regular cadence and constructing light curves 
of all of the sources within the survey region. Clearly an expanded field of view would determine the total number of sources that could be monitored.

2. Many low-mass stars (spectral types $\mathrm{K}$ and $\mathrm{M}$ ) show significant "radio activity," often with radio flares or bursts on short time scales [13]. This radio emission is thought to be linked to coronal processes on the stars, likely closely coupled to the magnetic field structure. Study of the coronal processes in these extreme cases may provide understanding of solar processes, which could impact not only astrophysics but aspects of the Earth-Sun connection as well. Most, if not all, of the strongly "radio active" stars in the solar neighborhood are known, but the typical separation on the sky is fairly large. Similar to the case for pulsar timing, monitoring a large number of low-mass stars for radio bursts would have a much higher throughput if access to a wide field of view becomes possible.

We emphasize that these are only two possible examples, chosen to illustrate the possible range of transient survey programs. The actual impact of the field of view on any transient program will also depend upon the temporal characteristics of the transients being targeted, their luminosity function, and distribution on the sky, to the extent that these parameters are known.

\section{References}

[1] Belloche, A., Garrod, R. T., Müller, H. S. P., Menten, K. M., Comito, C., \& Schilke, P. 2009, Astron. \& Astrophys. 499, 215.

[2] Belloche, A., Menten, K. M., Comito, C., Müller, H. S. P., Schilke, P., Ott, J., Thorwirth, S., \& Hieret, C. 2009, Astron. \& Astrophys. 482, 179.

[3] Bower, G. C. et al. 2008, Astrophys. J. 666, 346.

[4] C. L. Carilli and S. Rawlings, Science with the Square Kilometer Array, New Astron. Rev., 48, Elsevier, Amsterdam, 2004

[5] Fiedler, R. L., Dennison, B., Johnston, K. J., \& Hewish, A. 1987, Nature 326, 675.

[6] Gaensler, B. M. 2004, "Key Science Projects for the SKA,” SKA Memorandum 44

[7] Hallinan, G. et al. 2007, Astrophys. J. 663, L25.

[8] Hankins, T. H., Kern, J. S., Weatherall, J. C., \& Eilek, J. A. 2003, Nature 422, 141.

[9] Hyman, S. D. et al. 2005, Nature 434, 50.

[10] Manchester, R. N. et al. 2001, Mon. Not. R. Astron. Soc. 328, 17

[11] McLaughlin, M. A. et al. 2006, Nature 439, 817.

[12] Murphy, E. 2009, Astrophys. J., submitted

[13] Osten, R. A., \& Bastian, T. S. 2008, Astrophys. J. 674, 1078.

[14] Seymour, N., Huynh, M., Dwelly, T., et al. 2009, Mon. Not. R. Astron. Soc., in press; arXiv:0906.1817

[15] Snyder, L. E., Hollis, J. M., Jewell, P. R., Lovas, F. J., \& Remijan, A. 2006, Astrophys. J. 647, 412.

[16] Turnbull, M. C. ,\& Tarter, J. C. 2003, Astrophys. J. Supp. 145, 181. 